\title{
Helping blind mice to see the light
}

An algae-derived protein that can restore some measure of light sensitivity to mice made blind by retinal degeneration offers a promising first step toward a gene therapy strategy for treating certain types of blindness.

Retinal degeneration disorders, such as the heritable condition retinitis pigmentosa (RP), are leading causes of human blindness. An estimated 1 in 4,000 people worldwide have a genetic predisposition for $\mathrm{RP}$, in which genetic mutations result in loss of photoreceptor neurons in the retina. A suggested potential treatment is the transplantation of healthy cells, but this could entail complex procedures and the risk of host transplant rejection.

In new work published in Neuron (6 April), Wayne State University (Detroit, MI) researcher Zhuo-Hua Pan and his colleagues test another possibility-using gene therapy to introduce a foreign light-sensitive channel protein into the surviving, nonphotosensing neurons of the retina. These neurons normally help transmit the signals generated by photosensors to the brain, but when coaxed into producing the algaederived light-sensitive ion channel channelrhodopsin-2 (ChR2), seem to acquire limited photosensing capabilities.

Pan's team worked with a mouse model for RP, injecting their eyes with an adenoassociated virus construct that allows infected cells to produce ChR2. They found that RP mice, despite the lack of photosensing neurons, seemed to retain other types of retinal neurons, and that these were readily infected with virus. Neurons producing ChR2 showed substantial light sensitivity, generating action potentials in response to light from a reasonably broad range of visible wavelengths. Importantly, they also demonstrated that the treated RP mice generated visually evoked potentials (i.e., activity in the visual cortex of the brain) in response to light exposure.

This is only a beginning, of course. Among other issues, the light sensitivity is considerably weaker than that in wild-type retinas. Additionally, the cells expressing ChR2 currently show sensitivity only to increasing light intensity; this differs from healthy eyes,

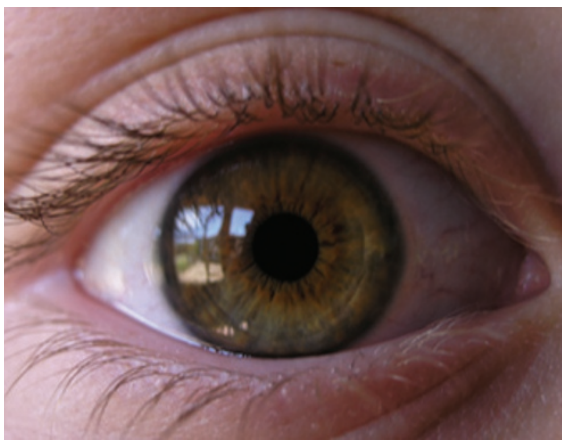

in which light sensation results from a balance between neuron classes that sense either increases or decreases in light levels. Pan hopes to address this difference in sensitivity in the near future, perhaps by targeting their virus to specific cell types. In the meantime he indicates that his team is now looking into assessing the impact of this treatment on the visual behavior of rodents - and, in the long term, larger mammalian animal models - to better understand the extent to which onceblind animals benefit from a little additional illumination.

Michael Eisenstein

\section{'ETHICAL' STEM CELISS FOUND_IN MOUUSE TESTIES}

Scientists have discovered a population of easily isolable stem cells with reprogrammable properties in the testes of adult male mice. If similar cells exist in humans, they might then provide a noncontroversial source of pluripotent cells for use in regenerative therapies.

Embryonic stem cells (ESCs) may be the key to curing various diseases, from diabetes to Parkinson's, because scientists can coax them to differentiate into any cell type. However, various religious groups contest their use because it is necessary to destroy embryos to obtain them. Indeed, laws in the United States strictly limit the use of federal funds in stem cell research.

In recent research, a group led by Gerd Hasenfuss of the Georg-August-University of Göttingen (Germany) found that mouse spermatogonial stem cells (SSCs), which normally become sperm cells, seem to act similarly to ESCs (Nature, published online 24 March, doi: 10.1038/nature04697). They isolated SSCs from the testes of transgenic mice and demonstrated the cells' ability to differentiate into various cell types in culture. Next, the German group tested the cells' behavior in vivo, with promising results. SSCs injected into mouse embryos contributed to development of various organs, including heart, brain, intestine, and lung. When injected into immunodeficient mice, the cells formed teratomas, much as ESCs do.

Kaomei Guan, first author on the paper, assured Lab Animal that men do have SSCs with self-renewal and differentiation potential, and said that "the ultimate goal is to make the technique work to reprogram [human] SSCs into ESC-like cells."

The use of similar techniques in humans would allow the cell therapy to be done using cells derived from the patient's own body via biopsy, solving the immunological problems associated with the use of foreign cells.

Guan noted that men would clearly benefit from such a discovery but that there is still hope for women. Recent research in mice revealed that female germline stem cells exist after birth, but no one has yet developed a culture system to test the cells' potential. "The chances of finding ESC-like cells in women is high," Guan told Lab Animal. The research team's next step is to isolate and reprogram female germline stem cells.

Anisa Abid 